\title{
A Simple Theoretical Comparison between Two Basic Schemes in Function of the Earth's North Pole Detection: The Static Method
}

\author{
G. Eduardo Sandoval-Romero and Víctor Argueta-Díaz \\ Centro de Ciencias Aplicadas y Desarrollo Tecnológico, Universidad Nacional Autónoma de México, \\ A. P. 70-186, 04510 Mexico, DF, Mexico \\ Correspondence should be addressed to G. Eduardo Sandoval-Romero, eduardo.sandoval@ccadet.unam.mx
}

Received 28 January 2010; Accepted 12 May 2010

Academic Editor: Hai Xiao

Copyright ( 2010 G. E. Sandoval-Romero and V. Argueta-Díaz. This is an open access article distributed under the Creative Commons Attribution License, which permits unrestricted use, distribution, and reproduction in any medium, provided the original work is properly cited.

The objective of this paper is to compare two gyrocompass' schemes for the detection of Earth's true north. These schemes are refered to as dynamic and static methods. We present a model to characterize the sensitivity of each model as a function of the output signal of the sensor element. We use a fiber optic gyroscope (FOG) as the sensor element.

\section{Introduction}

The design and implementation of navigation instruments was greatly improved by the appearance of the laser. The laser allowed the creation of systems that are less prone to be affected by natural external phenomena and at the same time to ease the fabrication. An example of one of these navigation instruments is what is known as Laser Ring Gyroscope (LRG) [1-5]. The appearance of optical fiber allowed for the implementation of fiber gyroscopes based on the principles of the LRG. The main advantages of fiber optic gyroscopes (FOG) [6-9] are the complete isolation of the system to external factors, the confinement of the optical path, the reduction of volume, the large sensitive area, and the high sensitivity that it possesses. These advantages make FOG devices highly reliable and efficient. It is not unusual to find FOGs as a core element in the implementation of gyrocompasses for navigation instruments $[10,11]$.

The further improvement, development, and design of navigation instruments are topics of actual interest in industry and academia [12]. The reduction in fabrication costs and dimensions allow for these instruments to be found in a variety of vehicles (e.g., automobiles, sailing ships) with different applications (e.g., heading, roll-over detectors). Gyrocompass' design and characterization depends on its use and application. It is necessary, therefore, to have a detailed analysis for the different gyrocompass configurations [1316]. Due to FOG's relevance in gyrocompass systems, we analyze the output signal of the FOG to characterize the device and propose recommendation for each scheme application.

In this paper, an analysis of the output characteristics of two basic gyrocompass schemes are compared. These two schemes are referred to as static and dynamic. In Section 2, we present the basic principles of gyrocompass' operation for earth's true north determination. In Section 3, we present the static scheme and its model. We present the dynamic scheme and model in Section 4. In Section 5, we present the FOG characteristic measurements of noise and scale factor coefficients. Finally, in Section 6 conclusions are presented.

\section{Determination of Direction to the True North}

The most important part in a gyrocompass for true Earth's north detector is, without doubts, the based FOG speed rotation sensor. This sensor determines the sensitivity characteristics of the gyrocompass. The operation principles of the FOG are analyzed in $[17,18]$.

To determine the direction of Earth's true north, an FOG is used where the output signal is proportional to the scalar multiplication of Earth's rotation vector with the FOG's sensitive vector $[13,14,19,20]$. Figures 1 and 2(a) 
show an FOG that is located on a tangential plane, called horizon plane hereafter, referenced to Earth with its sensitive axis parallel to the horizon plane. To take the appropriate measurements needed to determine the true north, the FOG will be rotating perpendicularly to the sensitive axis. The optical radius of the FOG corresponds to the radius of the contour or winding (see Figure 3); the direction of the radius is projected like the area of the fiber loop, located on the plane orthogonal to the angular velocity vector [7]. If the FOG rotation axis is aligned to the North-South axis (which is parallel to the Earth rotation axis), and if its horizon plane is tilted some degrees, similar to the local geographical latitude, then it is possible to measure the Earths' rotation speed, that is, $15.041 \mathrm{deg} / \mathrm{h}$. Figure 2 (b) shows the sinusoidal output signal for a gyroscope rotation around the vertical axis.

In general, for the determination of the true north or true meridian [21], it is necessary that the sensitivity vector, which is parallel to the horizontal plane, rotates (successively or in fixed angles) around the FOG's rotation axis. It is also necessary to take $i$-number of measurements, $U_{i}$, of the output signal voltage of the FOG. This output signal is proportional to the scalar product of Earth's rotation vector, $\Omega$, and the FOG sensitivity vector, $\bar{K}$, where the coordinates are created by the angles formed by the vector projections in a Cartesian plane $X Y Z$, so we have

$$
\begin{gathered}
\bar{K}=K\left(\cos \Theta_{a} \cdot \cos \Theta_{b}, \sin \Theta_{a}, \cos \Theta_{a} \cdot \sin \Theta_{b}\right), \\
U_{i}=K \Omega \cos \Theta \cos \Phi_{i}
\end{gathered}
$$

where, $\Theta$ is the geographical latitude point in the Earth where the measure is realized, $\Theta_{b}$ is the angle between $\bar{K}$ and the $X Z$ plane, $\Theta_{a}$ is the angle between $\bar{K}$ projected in the $X Z$ plane and the $X$ axis, $K$ is the FOG scale factor coefficient, $\Omega$ is the angular speed of the Earth (which has a value of $15.041 \mathrm{deg} / \mathrm{h}$ ), and $\Phi_{i}$ is the azimuth, which is the angle on the horizon plane between the true meridian up to the FOG's sensitivity vector projection on the horizon plane. To calculate the azimuth value it is necessary to take several readings, $U_{1}, U_{2}, \ldots, U_{n}$. How these measurements are taken distinguishes the static and dynamic methods.

The base of both models is the measurements in real time of the FOG characteristics. It is necessary to take into account the changes (either phase or intensity) of the FOG's output signal. Due to the constant Earth's speed of rotation, and with the knowledge of the latitude coordinate where the measurements are taken, the output signal of the gyroscope can then be modeled only with the output characteristics and the projections of the terrestrial rotation in the gyroscope sensibility axis. This model describes the real sign very well [21], but it is necessary to remember that this model is only an approximation; since it does not take into account the induced random errors from external factors: vibration and temperature fluctuation in the rotation process of the gyroscope in the platform.

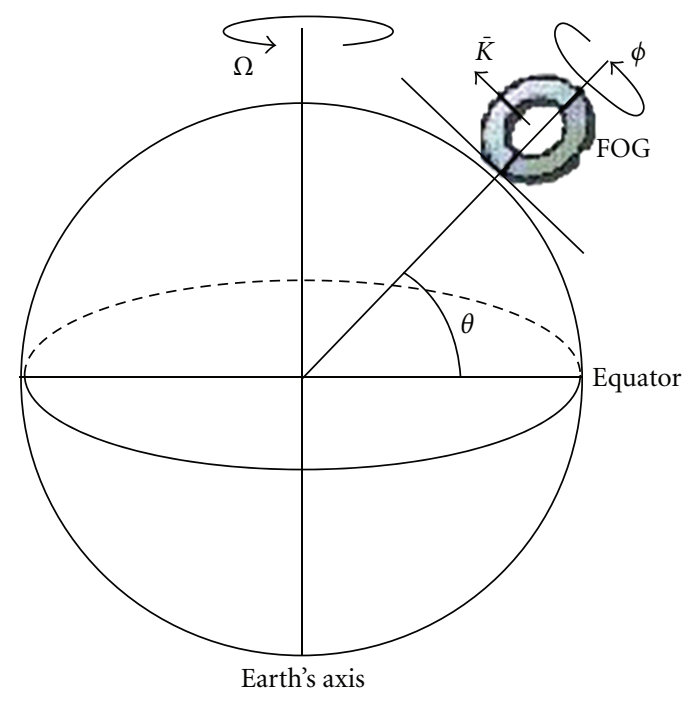

FIgURE 1: Guidance of the fiber optics gyroscope.

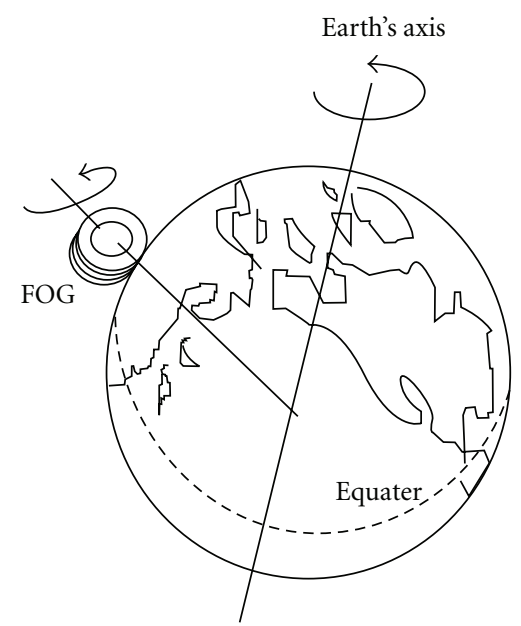

(a)

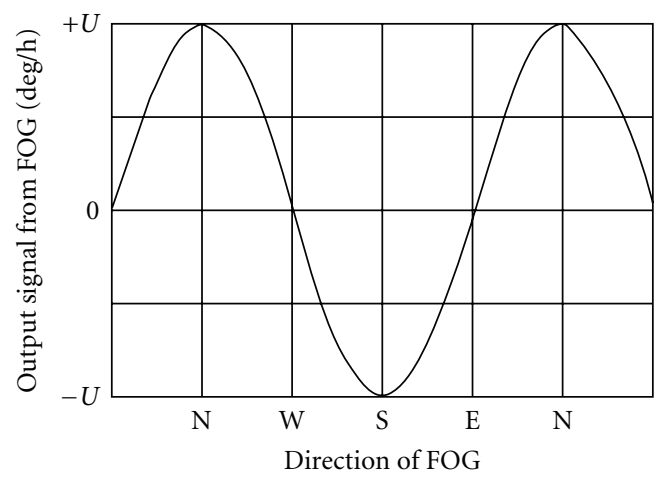

(b)

FIGURE 2: (a) A representation of the guidance of the FOG and the basic work operations gyrocompass, (b) typical output sign from FOG is shown in the oscilloscope display. 


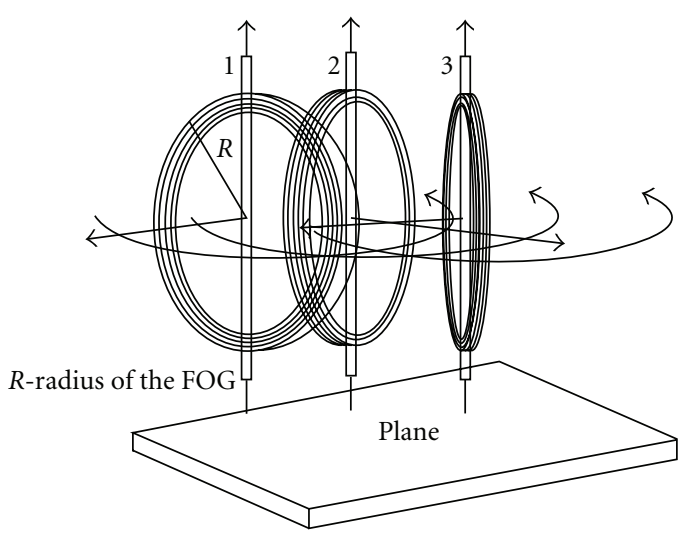

FIGURE 3: Schematic illustration of the static method.

\section{Static Method for the Determination of Earth's True North}

In this method, it is necessary to take three or more consecutive readings with the FOG's sensitivity vector at different positions $[13,14]$. This can be done by taking measurements while rotating the FOG along its axis at three or more positions, as shown in Figure 3. The direction toward the true North is determined through the signal processing of the FOG output at these points. The first measurement, $U_{1}$, will be our reference, and it is represented by (3)

$$
U_{1}=K \Omega \cos \Theta \cos \phi
$$

For the second measurement, we rotate the FOG $\Phi_{1}$ degrees from the first point.

For this case, the voltage measurement, $U_{2}$, can be calculated by

$$
U_{2}=K \Omega \cos \Theta \cos \left(\phi+\Phi_{1}\right) .
$$

In a similar way, a third measurement, $U_{3}$, has to be taken. This time we further rotate the FOG by $\Phi_{2}$ degrees, obtaining the equivalent sensor position measurement

$$
U_{3}=K \Omega \cos \Theta \cos \left(\phi+\Phi_{2}\right) \text {. }
$$

Then, in general

$$
\begin{aligned}
& U_{1}-U_{2}=K \Omega \cos \Theta\left[\cos \phi-\cos \left(\phi+\Phi_{1}\right)\right], \\
& U_{1}-U_{3}=K \Omega \cos \Theta\left[\cos \phi-\cos \left(\phi+\Phi_{2}\right)\right] .
\end{aligned}
$$

Assuming that $\Phi_{1}$ and $\Phi_{2}$ are known, then $\phi$ can be calculated by iterations.

If $\Phi_{1}=-\pi / 2$ and $\Phi_{2}=\pi / 2$, we have

$$
\frac{U_{1}-U_{2}}{U_{1}-U_{3}}=\frac{[\cos \phi+\sin \phi]}{[\cos \phi-\sin \phi]} \text {. }
$$

The resolution limit for this method can be calculated as follows:

$$
\Delta \Theta=\frac{180 \cdot \Delta \Omega}{\left(\pi \cdot U_{\max } \cdot \cos \Theta\right)}[\operatorname{deg}]
$$

where $U_{\max }$ is the maximum registered signal at the FOG output as calculated by equation (2). The FOG resolution, $\Delta \Omega$ is calculated as follows [17]:

$$
\Delta \Omega=\sqrt{n_{\mathrm{sn}}^{2}+n_{k}^{2}+n_{\mathrm{DR}}^{2}+n_{\mathrm{br}}^{2}} \cdot\left(\frac{h c^{2}}{2 \pi e q R l P_{\mathrm{tot}}}\right) \cdot\left(\frac{180}{\pi}\right)[\mathrm{deg} / \mathrm{s}],
$$

where, $n_{\mathrm{sn}}^{2}$ is the shot noise sign, $n_{k}^{2}$ is the sign of noise induced by the Kerr effect, $n_{\mathrm{DR}}^{2}$ is the sign of noise induced by the Rayleigh dispersion effect, $n_{\mathrm{br}}^{2}$ is the sign of noise induced by the birefringence effect; $q$ is the quantum efficiency of the photodetector, $e$ is the unitary electron charge, $R$ is the optic ring radius (contour), $l$ is the fiber optic ring longitude, $h$ is the Plank's constant, $c$ is the speed of light in the vacuum, and $P_{\text {tot }}$ is the optical power input in the fiber optic.

\section{Dynamic Method of North Determination}

Contrary to the static method, in the dynamic method the sensitive axis of the FOG is in constant movement, and this can be achieved by rotating the FOG at constant angular speed [22]. The output signal is, therefore, a sinusoidal wave. As the FOG's position is modified, a change in phase can be detected. The output signal of the FOG is described by

$$
U=\Omega \sin \Theta
$$

where $\Omega$ is the Earth's angular speed rotation, and $\Theta$ is the direction of the optical radius of the FOG. Under this scheme, at a specific number of laps of the FOG, a measurement is taken. The reading is made during a finite time, $\tau$, and can be triggered by a mark or lap counter on the FOG rotational base. The azimuth's accuracy determination is then described by

$$
\Delta \Theta=\frac{(180 \cdot \Delta U)}{\left(\pi \cdot \Omega_{m} \cos \Theta\right)}[\operatorname{deg}] .
$$

Accuracy can be improved if Earth's rotation speed is calculated constantly and if the azimuth is calculated by a sinusoidal function. $\Delta U$ is the theoretical limit of the measurement's accuracy at each trigger event. It is limited by the trigger noise, $\Delta \Omega_{\mathrm{op}}$, and the resolution of the associate electronics, $\Delta \Omega_{\mathrm{el}}$. The trigger noise limit is determined by

$$
\begin{gathered}
\Delta \Omega_{\mathrm{op}}=\frac{180}{a \cdot \sqrt{\left(P_{r} q / e\right) \cdot \tau}}[\mathrm{deg} / \mathrm{s}], \\
a=\frac{4 \pi R l}{\lambda c},
\end{gathered}
$$

where $a$ is the scale factor of the FOG, $P_{r}$ is the detected optic power, $q$ is the quantum efficiency of the photodetector, $e$ is the unitary electron charge, $\tau$ is the trigger average time, $R$ is the optical ring radius (contour), $l$ is the optical ring longitude, $\lambda$ is the radiation source wavelength, and $c$ is the speed of light in vacuum.

Sensitivity can be enhanced by increasing the scale factor, that is, using a longer optical fiber. In this case, the 


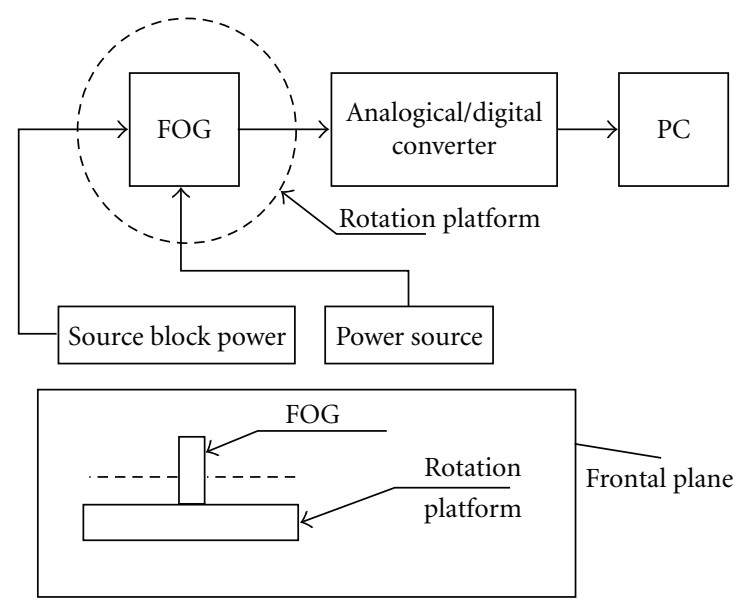

FIGURE 4: Schematic illustration of the experimental device.

detected optic power will decrease, and the longitude should be optimized. The electronic resolution is limited by the analogical-digital converter

$$
\Delta \Omega_{\mathrm{el}}=\frac{\Omega_{d}}{2^{b} T}
$$

where $\Omega_{d}$ is the dynamic range, $b$ is the $\mathrm{A} / \mathrm{D}$ converter resolution (bits), and $T$ is the number of measurements.

\section{FOG's Characteristics Measurements (Characteristic Noise and Factor Scale Coefficient)}

As it was mentioned previously, the study and construction of a gyrocompass is based on an FOG. For this reason, it is necessary to characterize the FOG performance. We are interested in the FOG's characteristic noise and the scale coefficient. We use the experimental configuration shown in Figure 4 to evaluate those parameters, which are independent of the scheme under which the gyrocompass is working. The measurements are carried out under the static scheme.

5.1. Characteristic Noise. The measured standard deviation (root mean square) is taken as the measurement error of the FOG's rotation speed, and it is represented by

$$
\sigma=\left(\frac{U_{i}^{2}-\bar{U}^{2}}{m(m-1)}\right)^{1 / 2}[\operatorname{deg} / \mathrm{h}]
$$

where $U_{i}$ is the $i$ value of the output voltage measurement, $\bar{U}$ is average value of the output voltage measurement, and $m$ is the quantity of measurements. The standard deviation can be reduced if we increase the bandwidth, $B$, of the measurement

$$
\sigma=\frac{1}{B}
$$

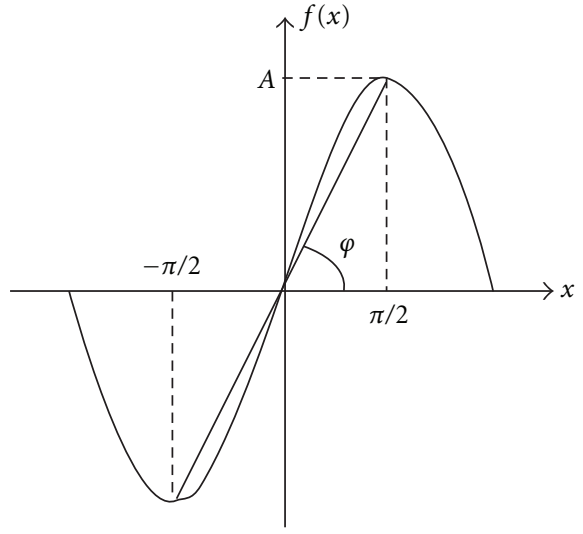

(a)

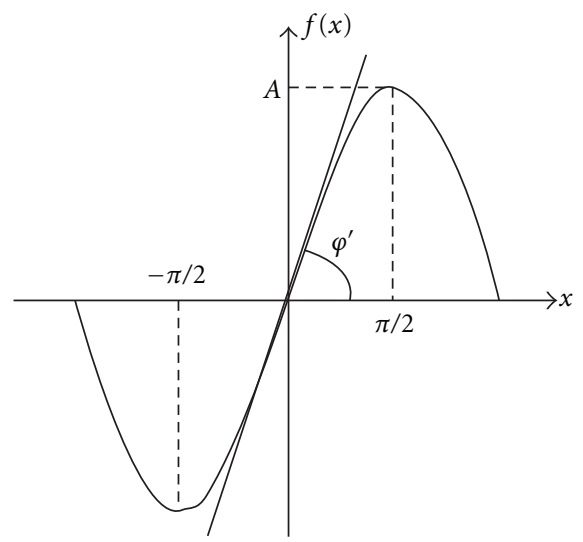

(b)

FIGURE 5: Output signal from gyroscope, (a) original signal, (b) new coordinates.

We know that $f=1 / t$; where $t$ is the time. For a particular $\sigma$ 's value, the characteristic noise at the filter's bandwidth is calculated by

$$
\sigma_{B}=\frac{\sigma}{\sqrt{B}}=\frac{[\operatorname{deg} / \mathrm{h}]}{\sqrt{\mathrm{Hz}}}=[\operatorname{deg} / \mathrm{h} / \sqrt{\mathrm{Hz}}]
$$

Besides white noise, the gyroscope output signal contains a component of low-frequency amplitude, which causes a drift in the gyrocompass. The drift is commonly measured in $\pm[\mathrm{deg} / \mathrm{h}]$. The noise and the drift play different roles depending on the gyroscope application. In stabilization and control systems it is important to have a low noise. For the navigation, the drift is the most important parameter.

5.2. Scale Factor Coefficient. To determine the scale factor coefficient, $K$, of the gyroscope [21], we use its output signal, shown in Figure 2(b), which has a sinusoidal form. Some calculation errors are due for the periodicity of functions like this one. Therefore, for the creation of an FOG with an acceptable dynamic interval, it is necessary to do a special treatment to its output sign. 
The following method was elaborated to find the scale factor coefficient depending on the FOG's location,

$$
K=\frac{U_{\max }-U_{\min }}{\Omega \cos \Theta}
$$

where $U_{\max }$ and $U_{\min }$ correspond to the investigated interval, that is, similar to half a period, with its corresponding phase between $(\pi / 2)$ and $(-\pi / 2)$.

To measure the real error of the Earths' rotation speed, it is necessary to obtain the coefficient relative only to a point with coordinates $(0,0)$ in our new coordinated system. We show this in the Figure 5, where we obtain

$$
\begin{gathered}
\operatorname{tg} \varphi=f^{\prime}(x)=\left.A \cos x \Longrightarrow(\operatorname{tg} \varphi)\right|_{x=0}=A \cos (0)=A \\
\varphi^{\prime}=\left.A \cos x\right|_{x=0}=A \\
\varphi=\operatorname{arctg} \frac{2 A}{\pi} \Longrightarrow \varphi=\operatorname{arctg} \frac{2 \varphi^{\prime}}{\pi}
\end{gathered}
$$

Having the condition that $\left|2 \varphi^{\prime} / \pi \ll 1\right|$, then we have that $\operatorname{tg}(x) \rightarrow x$.

So we obtain

$$
\begin{aligned}
\varphi=\frac{2 \varphi^{\prime}}{\pi} & \Longrightarrow \varphi^{\prime}=\frac{\pi \varphi}{2}, \\
\varphi^{\prime} & \approx \frac{\pi}{2} \varphi .
\end{aligned}
$$

We assign the variables $K_{0}=\varphi^{\prime}$ and $K=\varphi$; we obtain the final expression to determine the coefficient of the scale factor.

$$
K_{0}=1.57 K
$$

\section{Concluding Comments}

Taking into consideration the previously exposed wok, we can conclude the following.

(a) The use of the static method allows diminishing the demands of mechanical fixture that are present in the gyrocompass, because all the measurements are carried out in static state and therefore, the vibrations, in the rotational platform, does not affect the FOG output signal. One of its disadvantages is the increase in measurement time, due to the rotation time of the gyroscope from one point to another, longer stabilization time. For the use of this method, it is necessary to provide a mathematical model able to solve the system of previously described equations, in real time.

(b) The dynamic method allows carrying out continuous measurements of the true north direction, with an autotuner system. The dynamic method allows to diminish low-frequency (drift) with efficiency, but these advantages are possible only with a complex mechanical fixture; a very important disadvantage, is that when diminishing the vibration of the rotational platform, in its frequency, the synchronized detector cannot filter this signal of the gyroscope measured signal.

(c) The election of the gyrocompass depends on the FOG characteristics; especially if an FOG with high sensibility
$(<0.1 \mathrm{deg} / \mathrm{h})$ and very low drift is required. It is preferable to use the prototype with static model. Now, if an FOG is used with low sensibility (within a $1 \mathrm{deg} / \mathrm{h}$ order) and with a sufficiently big value of the drift, it is better to use the prototype with dynamic model, which allows to carry out a gyrocompass with the accuracy of the order of some dozens of degrees.

The final election of the FOG and the gyrocompass prototype state is analyzing the costs of all the prototype elements (mechanic and electronic), and also the required accuracy of the gyrocompass.

\section{Acknowledgment}

This paper was supported by grant J50594 Consejo Nacional de Ciencia y Tecnología-México.

\section{References}

[1] C. V. Heer, "Interference of electromagnetic and matter waves in a nonpermanent gravitational field," Bulletin of the American Physical Society, vol. 6, p. 58, 1961.

[2] A. H. Rosenthal, "Regenerative circulatory multiple-beam interferometry for the study of light-propagation effects," Journal of the Optical Society of America, vol. 52, no. 10, pp. 1143-1147, 1962.

[3] W. M. MacEk and D. T. M. Davis Jr., "Rotation rate sensing with traveling-wave ring lasers," Applied Physics Letters, vol. 2, no. 3, pp. 67-68, 1963.

[4] J. M. Catherin and B. Dessus, "3.1-Traveling-wave laser gyrocompass," IEEE Journal of Quantum Electronics, vol. 3, no. 11, pp. 449-453, 1967.

[5] A. Lawrence, Modern Inertial Technology: Navigation, Guidance and Control, Springer, Berlin, Germany, 2nd edition, 1998.

[6] H. Lefèvre, The Fiber-Optic Gyroscope, Artech-House, Boston, Mass, USA, 1993.

[7] E. I. Yakubovich and G. B. Malykin, "The effect of noncoplanar winding of an optical fiber in a fiber ring interferometer on the phase difference of counterpropagating waves," Radiophysics and Quantum Electronics, vol. 45, no. 11, pp. 895-905, 2002.

[8] R. A. Bergh, H. C. Lefevre, and H. J. Shaw, "Overview of fiberoptic gyroscopes," Journal of Lightwave Technology, vol. 2, no. 2, pp. 91-107, 1984.

[9] B. Culshaw and I. P. Giles, "Fibre optic gyroscopes," Journal of Physics E, vol. 16, no. 1, pp. 5-15, 1983.

[10] H. Kajioka, T. Kumagai, H. Nakai, H. Iizuka, M. Nakamura, and K. Yamada, "Fiber optic gyro productization at Hitachi (invited paper)," in Fiber Optic Gyros: 15th Anniversary Conference, vol. 1585 of Proceedings of SPIE, pp. 17-22, 1991.

[11] A. Cordova, R. A. Patterson, E. L. Goldner, and D. Rozelle, "Interferometric fiber optic gyroscope with inertial navigation performance over extended dynamic environments " in Fiber Optic and Laser Sensors XI, vol. 2070 of Proceedings of SPIE, pp. 164-180, Boston, Mass, USA, 1993.

[12] S. M. Bennett, R. Dyott, D. Allen, J. Brunner, R. Kidwell, and R. Miller, "Fiber optic rate gyros as replacements for mechanical gyros," in American Institute of Aeronautics and Astronautics, pp. 1-7, 1998, AIAA-98-4401.

[13] R. B. Dyott and D. E. Allen, "A fiber optic gyroscope north finder," in Proceedings of the 10th Optical Fiber Sensors Conference, p. 442, Glasgow, Scotland, 1994. 
[14] R. B. Dyott, "Method for finding true north using a fibre-optic gyroscope," Electronics Letters, vol. 30, no. 13, pp. 1087-1088, 1994.

[15] T. Kumagai, N. Ashizuka, H. Nakai, and H. Kajioka, "Development of an optical gyrocompass using a high-sensitivity fiber optic gyroscope," in Fiber Optic and Laser Sensors X, vol. 1795 of Proceedings of SPIE, pp. 105-117, Boston, Mass, USA, September 1992.

[16] H. Kajioka, N. Ashizuka, T. Kumagai, and H. Nakai, "Experimental investigation on direction sensor using a fiber optic gyroscope with high resolution," in Proceedings of the 8th Optical Fiber Sensors Conference, pp. 38-41, 1992.

[17] G. E. Sandoval-Romero and V. A. Nikolaev, "Límite de detección de un giroscopio de fibra óptica usando una fuente de radiación superluminiscente," Revista Mexicana de Fisica, vol. 49, no. 2, pp. 155-165, 2003.

[18] B. Culshaw, "The optical fibre Sagnac interferometer: an overview of its principles and applications," Measurement Science and Technology, vol. 17, no. 1, pp. R1-R16, 2006.

[19] T. Tanaka, Y. Igarashi, M. Nara, and T. Yoshino, "Automatic north sensor using a fiber-optic gyroscope," Applied Optics, vol. 33, no. 1, pp. 120-123, 1994.

[20] T. Gaiffe, Y. Cottreau, N. Faussot, et al., "Highly compact fiber optic gyrocompass for applications at depths up to 3000 meters," in Proceedings of the International Symposium on Underwater Technology (UT '00), pp. 155-160, Tokyo, Japan, May 2000.

[21] I. Kim, Y. Kim, I. A. Matisov et al., "Dynamic scheme north finder using a fiber optic gyroscope," in Fiber Optic Sensors V, vol. 2895 of Proceedings of SPIE, pp. 228-236, Beijing, China, November 1996.

[22] V. E. Strigalev, I. A. Matisov, V. A. Nikolaev, and Yu. V. Ivanov, "Causes of the errors of a dynamic fiber-optic gyrocompass," Journal of Optical Technology, vol. 64, no. 7, pp. 657-660, 1997. 

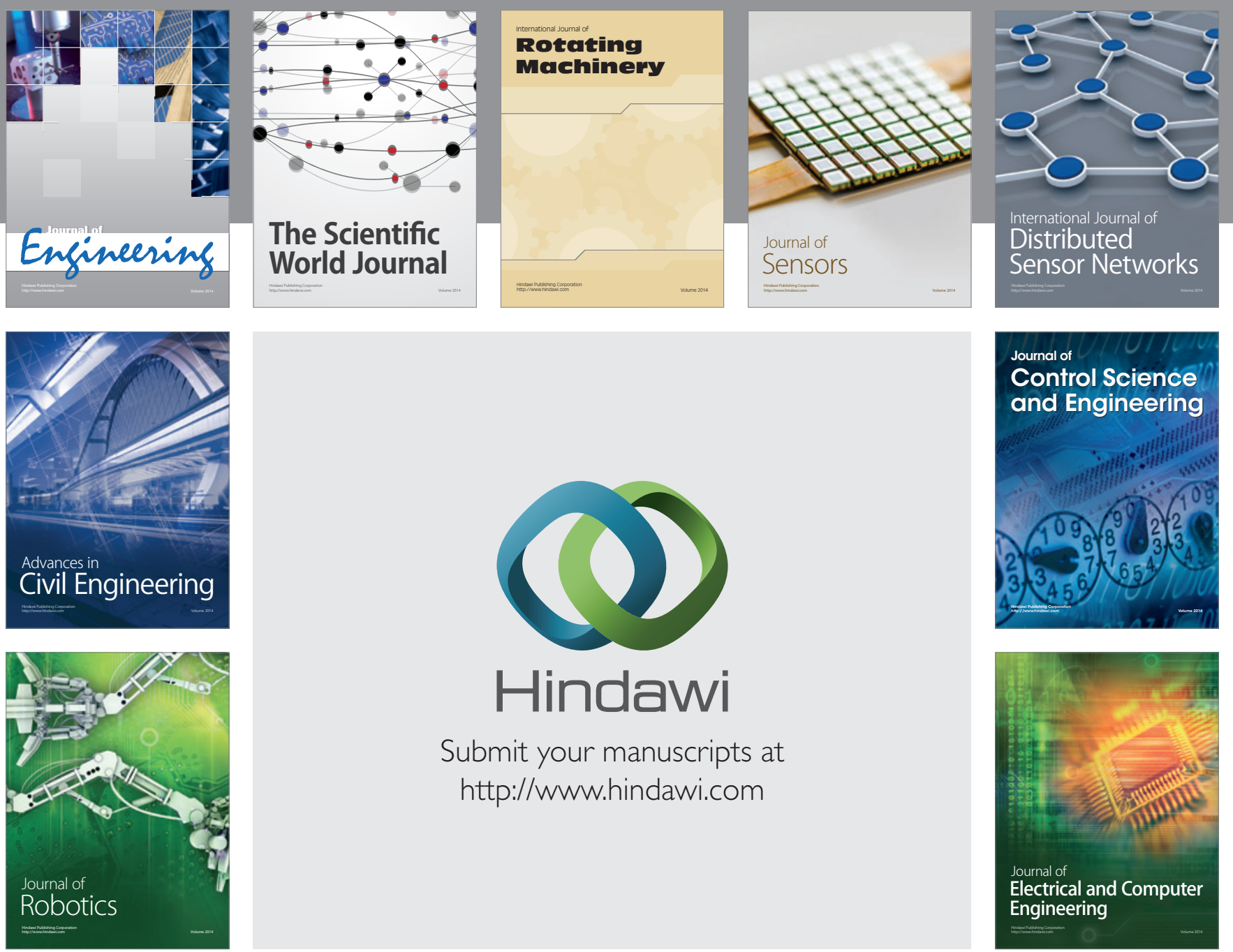

Submit your manuscripts at

http://www.hindawi.com
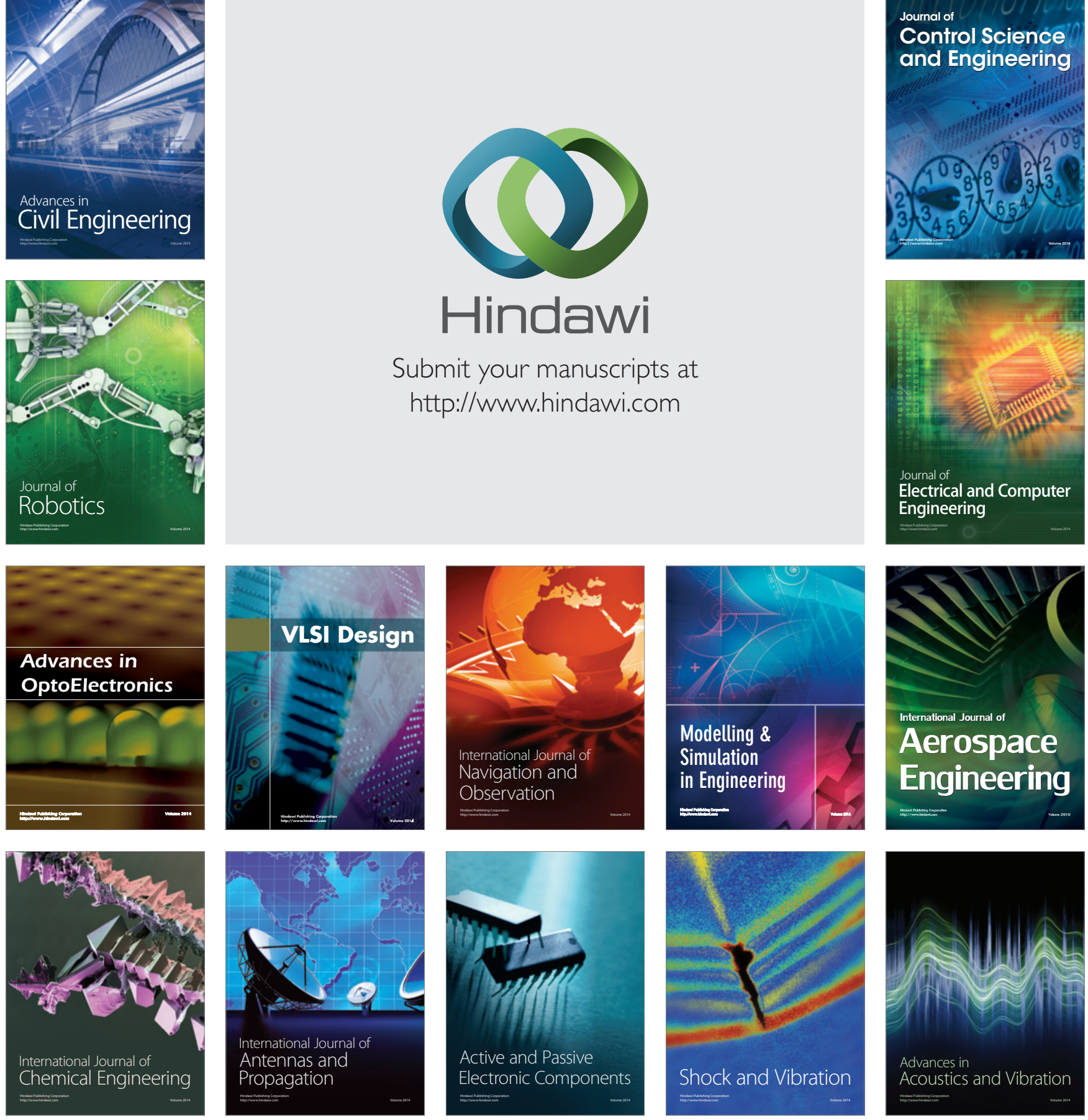